\title{
Mir-382 Promotes Differentiation of Rat Liver Progenitor Cell WB-F344 by Targeting Ezh2
}

\author{
Yongxia Zheng ${ }^{\mathrm{a}, \mathrm{b}}$ Jiansheng Zhou ${ }^{\mathrm{c}}$ Xuebo Lia, Guangtao Xu ${ }^{\mathrm{b}}$ \\ Mingliang Jin ${ }^{c}$ Ruilin Shen ${ }^{d}$ Ruibing Su ${ }^{a}$ Shuyu Zhan ${ }^{b}$ Baoyue Ding ${ }^{b}$ \\ Mingguang $\mathrm{Jia}^{f}$ Yuzhong Cuif ${ }^{f}$ Xiaojun $\mathrm{Yu}^{\mathrm{a}}$
}

${ }^{a}$ Department of Forensic Medicine, Shantou University Medical College, Shantou, Guangdong ${ }^{b}$ Medical College, Jiaxing University, Jiaxing, Zhejiang, 'The 92th Hospital of PLA, Nanping, dDepartment of Biochemistry and Diabetes Institute, Jiaxing Hospital of Traditional Chinese Medicine, Jiaxing University, Jiaxing, eKey Laboratory of Evidence Identification in Universities of Shandong Province, Shandong University of Political Science and Law, Jinan, Shandong, 'Linzi District People's Hospital, Zibo,

Shandong, China

\section{Key Words}

Hepatic differentiation • MiR-382 • Ezh2

\begin{abstract}
Background/Aims: Liver progenitor cells (LPCS) were considered as a promising hepatocyte source of cell therapy for liver disease due to their self-renewal and differentiation capacities, while little is known about the mechanism of LPC differentiate into hepatocytes. This study aims to explore the effect of miR-382, a member of Dlk1-Dio3 microRNA cluster, during hepatic differentiation from LPCs. Methods: In this study, we used rat liver progenitor cell WB-F344 as LPC cell model and HGF as inducer to simulate the process of LPCs hepatic differentiation, then microRNAs were quantified by qPCR. Next, WB-F344 cell was transfected with miR-382 mimics, then hepatocyte cell trait was characterized by multiple experiments, including that periodic acid schiff staining and cellular uptake and excretion of indocyanine green to evaluate the hepatocellular function, qPCR and Western Blotting analysis to detect the hepatocyte-specific markers (ALB, Ttr, Apo E and AFP) and transmission electron microscopy to observe the hepatocellular morphology. Moreover, Luciferase reporter assay was used to determine whether Ezh2 is the direct target of miR-382. Results: We found that miR-382 increased gradually and was inversely correlated with the potential target, Ezh2, during WBF344 hepatic differentiation. In addition, functional studies indicated that miR-382 increased the level of hepatocyte-specific genes. Conclusions: This study demonstrates that miR-382 may be a novel regulator of LPCs differentiation by targeting Ezh2.

Y. Zheng and J. Zhou contributed equally to this work.

\begin{tabular}{ll}
\hline Prof. Xiaojun Yu & Department of Forensic Medicine, Shantou University Medical College \\
& 22 Xinling Rd., Shantou 515041, GD (China) \\
& E-Mail xjyu@stu.edu.cn
\end{tabular}
\end{abstract}

\section{KARGER}




\section{Cellular Physiology Cell Physiol Biochem 2018;48:2389-2398

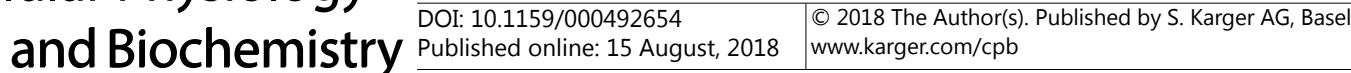

Zheng et al.: miR-382 Promote Hepatic Differentiation by Targeting Ezh2

\section{Introduction}

Liver progenitor cells (LPC), also termed oval cells, are liver-specific stem cells with the ability of self-renewal and differentiation into hepatocytes and cholangiocytes [1]. Despite quiescent in healthy liver, LPC was activated and proliferated in chronic liver disease [2$4]$, and the degree of LPC activation corresponds with the severity of liver fibrosis and inflammation [5]. Recent studies suggest that LPC represent a potential cell source of liver cell therapy due to their self-renewal and differentiation capacities [6]. However, one of the major hurdles of LPC therapies is the low differentiation efficiency, resulting in low yields of functional hepatocytes [7]. Therefore, it is necessary to understand the mechanism of LPC differentiate into hepatocytes and identify a novel pathway that could be therapeutically targeted.

microRNAs are small noncoding RNAs that modulate a variety of biological processes, including cellular differentiation, metabolism, and proliferation [8]. Interestingly, miRNA genes tend to occur in clusters [9], alluding to its evolutionary and functional implications. For example, Dlk1-Dio3 microRNA cluster is one of the largest miRNA clusters in the genome and located in the imprinted region on mouse chromosome 12qF1[10]. Multiple studies demonstrate that activation of the imprinted Dlk1-Dio3 region correlates with pluripotency levels of mouse stem cells [11] and a loss of the imprinted Dlk1-Dio3 region results in developmental abnormalities and fetal lethality [12], implicating their potential role in development. Actually, many of these miRNAs are differentially expressed in several adult stem cells and are related to the differentiation of several cell lineages. For example, miR300 regulates differentiation of primitive cardiac progenitors [13], Dlk1-Dio3 ncRNA reduce neural differentiation potential [14]. Besides, Cui L [15] reported that many of Dlk1-Dio3 miRNAs (miR-127, miR-376a, miR-136, miR-539, miR-154) were induced during hepatic differentiation of human umbilical cord lining-derived mesenchymal stem cells. While few reports have been published on the role of Dlk1-Dio3 microRNA clusters in LPC hepatic differentiation. Therefore, this study aims to explore the relationship between Dlk1-Dio3 microRNA cluster and LPC hepatic differentiation.

Polycomb group proteins initiate and maintain gene silencing through chromatin modifications and contribute to the maintenance of self-renewal in a variety of stem cells [16]. Enhancer of zeste homolog2 (Ezh2), a transcriptional factor, is the functional enzymatic component of Polycomb Repressive Complexes 2 (PRC2) [17], which initiate gene silencing by methylating histone $\mathrm{H} 3$ lysine 27 (H3K27me3). Generally, H3K27me3 is a repressive histone modification [18], which marks promoters of the developmental genes [19]. Recent studies demonstrate that Ezh2 is essential for expansion of hepatic progenitor population, and its loss of function result in decreased expression of liver-function related genes [20]. Accidently, bioinformatic analysis showed that several microRNAs from Dlk1-Dio3 cluster potentially target Ezh2. Thus, we hypothesized that some Dlk1-Dio3 microRNA may be involved in LPCs hepatic differentiation by targeting Ezh2.

In the present study, we mainly focused on miR-382, member of Dlk1-Dio3 microRNA cluster, based on its expression pattern during WB-F344 hepatic differentiation and its inducing hepatic differentiation, along with its target gene. our data suggests that miR-382 might also be a potential "initial" signal during hepatic differentiation.

\section{Materials and Methods}

\section{Cell culture and WB-F344 hepatic differentiation}

The WB-F344 rat hepatic oval cell line was obtained from the Fuxiang Biology Company, Shanghai, China. BRL-3A was obtained from the Institute of Biochemistry and Cell Biology, Shanghai, China. WB-F344 and BRL-3A cells were cultured in DMEM (Hyclone, Utah, USA) with 10\% fetal bovine serum fetal bovine serum (FBS, Sijiqing, Hangzhou, China) in a humidified atmosphere containing $5 \% \mathrm{CO}_{2}$ at $37 \mathrm{C}^{\circ}$. WB-F344 cells were seeded into 6 cell culture plates. 8h later, the HGF (Catalog: 315-23, PeproTech, USA) differentiation 


\section{Cellular Physiology Cell Physiol Biochem 2018;48:2389-2398

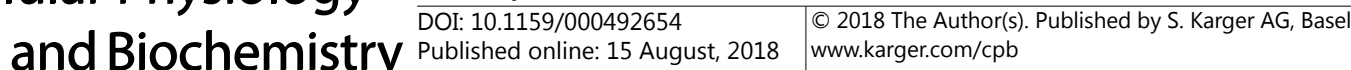

Zheng et al.: miR-382 Promote Hepatic Differentiation by Targeting Ezh2

culture medium $\quad(50 \mathrm{ng} / \mathrm{mL}$ HGF added to DMEM with $15 \%$ FBS) replaced the basal culture medium and continued culturing WB-F344 for 5 days. 5 days after treatment, $10 \mathrm{ng} / \mathrm{mL}$ HGF culture medium was used to keep differentiation for 20 days.

\section{Cell transfection}

miR-382 mimics, negative control sequences, Ezh2 siRNA, and control siRNA were obtained from Gene Pharma (Shanghai, China). Transfections were performed using a lipofectamine 2000 kit (Invitrogen, Carlsbad, CA) according to the manufacturer's instructions. RNA oligonucleotides were introduced into WB-F344 cells at a final concentration of 50nM.

Table 1. The primer sequences used

\begin{tabular}{lll}
\hline Gene & Forward primer & Reverse primer \\
\hline GAPDH & 5'-AGAAGGCTGGGGCTCATTTG-3' & 5'-AGGGGCCATCCACAGTCTTC-3' \\
Ttr & 5'-TCTCAAAGAGAATCAGCAGGTTTG-3' & 5'-CAGAGGAGGAACAGGCGAAG-3' \\
ApoE & 5'-GCTGACAGCTTTTCCTTCCG-3' & 5'-CAGTACCGTCAGTTCCTGTGT-3' \\
ALB & 5'-GTGAGCGAGAAGGTCACCAA-3' & 5'-CCTTGCAACACTTGTCCACG-3' \\
AFP & 5'-ACCATCGAGCTCGGCTATTG-3' & 5'-CGTTTCAGACTGGGAGCACT-3' \\
Ezh2 & 5'-GCACACTGCAGAAAGATCCA-3' & 5'-AGGTAGCACGGACACTGCTT-3' \\
Ezh2-UTR & 5'-GCCTCGAGCATCTACTACCTCTTC -3' & 5'-GCGAGCTCTGTTCAAGTTCATAA -3' \\
Mu-Ezh2 & 5'-CAAACTTTTATTGCCTTCTCA -3' & 5'-CTTTTAAAACATTACTATAAATTA -3' \\
\hline
\end{tabular}

Table 2. List of antibody and conditions

\begin{tabular}{lll}
\hline Antibody & Source & Catalog No \\
\hline GAPDH & Cell Signaling Technology (CST), USA & $2118 \mathrm{~s}$ \\
Actin & Cell Signaling Technology (CST), USA & 3700 \\
AFP & Arigo, Taiwan, China & ARG56134 \\
Ezh2 & Cell Signaling Technology (CST), USA & 5246 \\
H3K27me3 & Cell Signaling Technology (CST), USA & 9733 \\
Secondary antibody & SinoBio, Shanghai, China & AB501 \\
\hline
\end{tabular}

Periodic acid Schiff (PAS) staining

PAS staining was performed using PAS Staining Kit (Sigma-Aldrich, St. Louis, MO, USA). Briefly, cells were fixed by $4 \%$ paraformaldehyde for $30 \mathrm{~min}$ and then washed with $\mathrm{H}_{2} \mathrm{O}$. Then, they were oxidized in $0.5 \%$ periodic acid for $15 \mathrm{~min}$ at room temperature, rinsed in $\mathrm{H}_{2} \mathrm{O}$ for 1 min and treated with Schiff's reagent for $30 \mathrm{~min}$ at room temperature. After rinsing in $\mathrm{H}_{2} \mathrm{O}$ for $2 \mathrm{~min}$, counterstaining was performed with hematoxylin for $2 \mathrm{~min}$, rinsed in running tap water and observed under an inverted microscope.

\section{Cellular uptake and excretion of indocyanine green}

Indocyanine Green (ICG) (Catalog: 3599-32-4, Sigma-Aldrich, St Louis, MO, USA) was dissolved in DMSO at $100 \mathrm{mg} / \mathrm{mL}$, then added DMEM medium with the final concentration of $1 \mathrm{mg} / \mathrm{mL}$. After incubation at $37 \mathrm{C}^{\circ}$ for $60 \mathrm{~min}$, the medium with ICG was discarded and the cells were washed with PBS. Then, PBS was replaced by the culture medium and the cells were incubated at $37 \mathrm{C}^{\circ}$ for $6 \mathrm{hr}$. The excretion of ICG was examined again by microscopy.

\section{Transmission electron microscopy}

WB-F344 cells were fixed with $2 \%$ glutaraldehyde cacodylate buffer for 30 min and post-fixed with $1 \%$ osmium tetroxide for $30 \mathrm{~min}$, after which they were dehydrated through a series of graded ethanol. Samples were embedded in Epok 812 (Oken Shoji, Tokyo, Japan), and ultrathin sections were cut with an ultramicrotome (MT7000-Ultra, RMC, Tucson, AZ). Samples were stained with uranyl acetate and lead citrate, and were then viewed with an electron microscope (H-7500; Hitachi, Tokyo, Japan).

\section{Reverse transcription reaction and quantitative real-time PCR}

Total RNAs were extracted using Trizol reagent (Invitrogen, Mason, USA). The first-strand cDNA was generated using the Reverse Transcription System Kit (Invitrogen, Mason, USA). Reverse transcription was executed using the mi DETECT miRNA RT Kit (Ribobio, Guangzhou, China) for miRNA and PCR were performed using mi DETECT miRNA PCR Kit (Ribobio, Guangzhou, China) according to the manufacturer's protocol. U6 was used as internal microRNA control. Real-time PCR was performed using a standard SYBRGreen PCR kit on a Step One Plus system (Applied Biosystems 7300). GAPDH was used as an endogenous control to normalize the amount of total mRNA in each sample. All sequences are listed in Table 1. 


\section{Cellular Physiology Cell Physiol Biochem 2018;48:2389-2398 \begin{tabular}{l|l|l} 
and Biochemistry & Published online: 15 August, 2018 & $\begin{array}{l}\text { C 2018 The Author(s). Published by S. Karger AG, Basel } \\
\text { www.karger.com/cpb }\end{array}$ \\
\hline
\end{tabular}}

Western blot analysis

Total cell lysate was prepared in $1 \times$ SDS buffer. Proteins at the same amount were separated on SDS-PAGE, then transferred onto nitrocellulose membranes (Millipore, MA, USA). After incubation with antibodies specific for either AFP, Ezh2, H3k27me3 or GAPDH (Table 2), the blot was developed using goat anti-rabbit secondary antibody and visualized using enhanced chemiluminescence (Pierce, Rockford, USA).

\section{Luciferase reporter assay}

The 3'UTR of Ezh2 containing the miR-382 response element was cloned into the Xho I/Sac I site of pmirGLO control luciferase vector (Promega, Madison, WI). A mutant 3'UTR of Ezh2 was synthesized using a site-directed mutagenesis kit (Takara, Toyko, Japan). Primers are listed in Table 1.

WB-F344 cells were co-transfected with 500ng WT-Ezh2-pmirGLO/Mu-Ezh2 -pmirGLO constructs and miR-382 mimics or the negative control in a 24-well plate. Each sample was co-transfected with $50 \mathrm{ng}$ renilla luciferase control plasmid (pGL4.7) to monitor the transfection efficiency (Promega, Madison, USA). $48 \mathrm{~h}$ later, luciferase activity assay was performed using the dual luciferase reporter assay system (Promega, Madison, USA).

\section{Statistical analysis}

Data are expressed as means \pm standard deviation from three independent experiments. Data were analyzed by using $t$-test, ANOVA as indicated in the figure legends. All $p$ values were two-sided and obtained by using the SPSS 13.0 software package. $p<0.05$ was indicative of a statistically significant result.

\section{Results}

\section{HGF induce WB-F344 hepatic differentiation}

HGF has been implicated as an important factor in stimulating hepatic differentiation and HGF was widely used to trigger onset of hepatic differentiation from LPCs [21-23]. To induce rat liver progenitor cell WB-F344 differentiate into hepatocyte cell, we cultured WB-F344 cells in hepatic differentiation medium with HGF (described in "Materials and methods"). Briefly, WB-F344 cells were cultured in DMEM with $50 \mathrm{ng} / \mathrm{ml} \mathrm{HGF}$ in the first 5 days, then $\mathrm{HGF}$ was reduced to $10 \mathrm{ng} / \mathrm{ml} \mathrm{HGF}$ in the following days to maintain the differentiation.

Fig. 1. HGF induce WB-F344 differentiate into hepatocyte cells. A. Procedure of WB-F344 hepatic differentiation induced by HGF. B. WB-F344 shows epithelial morphology. Cytoplasmic accumulation of glycogen was determined by PAS staining, and intake of ICG in WB-F344 cells. All scale bars: 100um. C. Expression of the indicated genes was measured by Western Blotting analysis during HGF induced WB-F344 cells. D. Statistical graph of Western Blotting bands. Data are expressed as mean $\pm \mathrm{SD}$ of three repeat experiments. E. Expression of the indicated genes was measured by RT-PCR analysis during HGF induced WB-F344 cells. F. SEM of WB-F344 or WB-F344 treated 24h

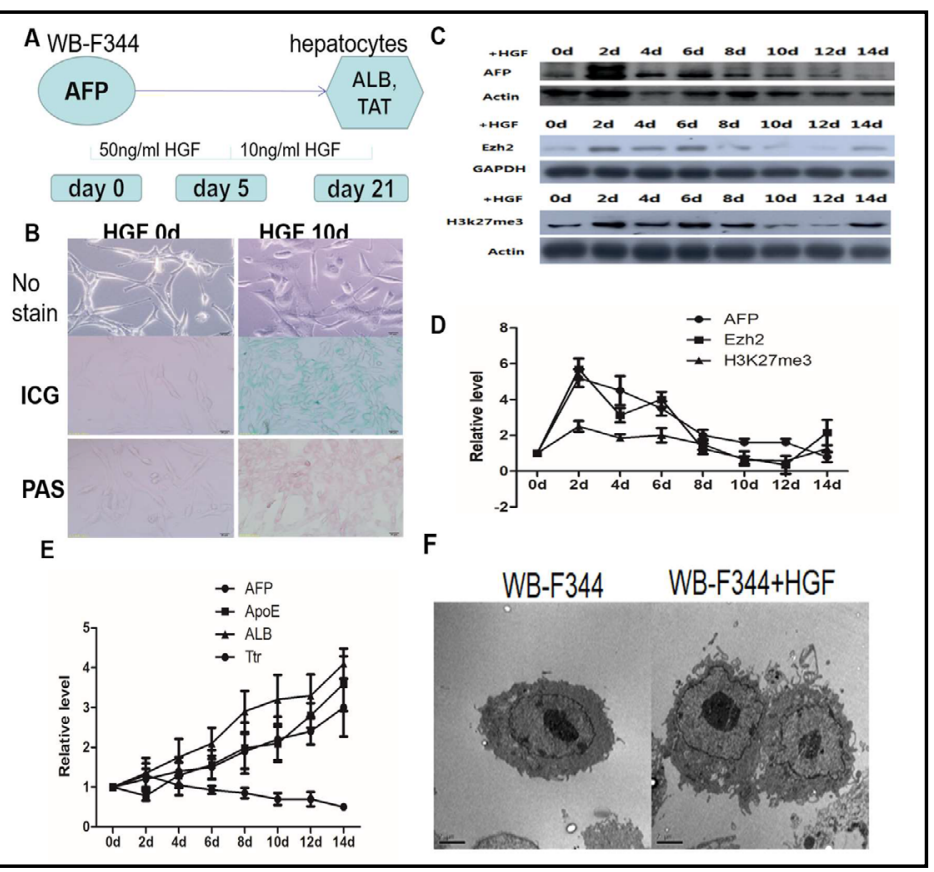
with HGF. 


\section{Cellular Physiology Cell Physiol Biochem 2018;48:2389-2398 \begin{tabular}{l|l|l} 
and Biochemistry Published online: 15 August, 2018 & $\begin{array}{l}\text { @) } 2018 \text { The Author(s). Published by S. Karger AG, Basel } \\
\text { www.karger.com/cpb }\end{array}$ \\
\hline
\end{tabular}

Contrarily, WB-F344 cells cultured in DMEM without HGF was used as control.

After culturing for 10 days, to confirm the validity of our hepatic differentiation model, several assays were performed to prove that the HGF-treated WB-F344 became hepatocytes ultimately. PAS staining of HGF-treated WB-F344 cells showed significant storage as mature hepatic cells (Fig. 1B). Besides, most HGF-treated WB-F344 cells were positive for ICG, while the control group were negative. Moreover, RT-PCR analysis revealed that robust upregulation of mature hepatocyte-specific transcripts (ALB, Ttr, Apo E) and decreased expression of hepatic stem cells markers (AFP) in WB-F344 cells at 12 days (Fig. 1D). Additionally, ultrastructural analysis with electron microscopy showed typical hepatocytic organelles such as mitochondria, lysosomes, and glycogen granules (Fig. 1E). Altogether, these results clearly indicate that WB-F344 could differentiate into hepatocyte-like cells.

To confirm the protein level of Ezh2 during WB-F344 hepatic differentiation, western blotting analysis was performed. As shown in Fig. 1C, both Ezh2 and its catalytic product, H3K27me3, were increased in the first 2 days and decreased transiently in the following days during WB-F344 hepatic differentiation.

\section{MiR-382 was induced during WB-F344 cells hepatic differentiation}

In the initial study, many of Dlk1-Dio3 miRNAs (miR-127, miR-376a, miR-136, miR539, miR-154) were induced during hepatic differentiation of human umbilical cord liningderived mesenchymal stem cells [15]. Fig. 2A showed genomic organization of microRNA located in Dlk1-Dio3 cluster. (Adapted from MJ Cairns [24]). To determine whether Dlk1-Dio3 microRNA cluster is also altered in liver progenitor cell WB-F344 hepatic differentiation, we performed qPCR analysis. As shown in Fig. 2B, we found 5 miRNAs was remarkably increased, of which miR-382 potentially target Ezh2.

To verify whether miR-382 in mature hepatocyte cells is always higher than LPCs, we measured the expression levels of miR-382 in rat liver tissues and BRL-3A cell. As revealed in Fig. 2C, the expression level of miR-382 in rat liver tissues and BRL-3A cells were relatively higher than that in WB-F344 cells, suggesting that miR-382 is widely induced during hepatic differentiation.

Fig. 2. miR-382 among Dlk1Dio3 microRNA cluster was induced during WB-F344 cells hepatic differentiation. A.Genomic organization of $14 \mathrm{q} 32$ microRNA clusters neighboring the Dlk1Dio3 imprinted domain. This Fig. was adapted from MJ Cairns. B. The expression of Dlk1-Dio3 microRNA clusters were measured in WBF344 and HGF induced WB-F344 cells at different time points. Data are shown as means and standard deviations from three independent experiments. The data were subjected to ANOVA. C. miR-382 expression levels in rat liver tissue, BRL-3A and WB-F344 cells by qRTPCR analysis. MiR-382 levels were

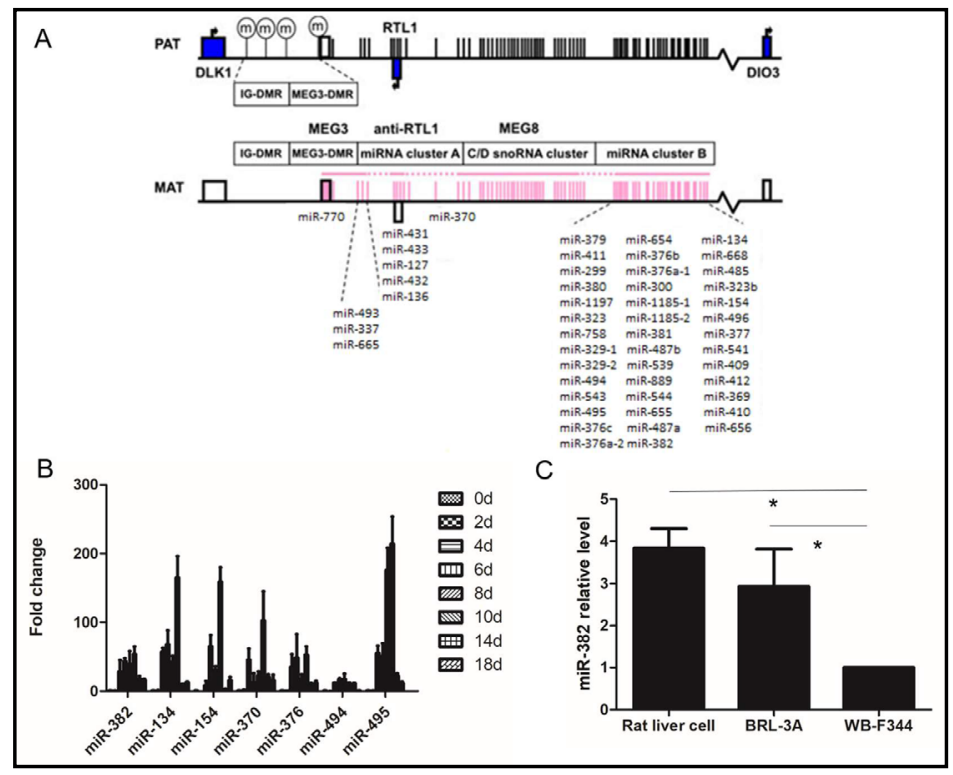
normalized to that of U6. Data are shown as means and standard deviations from three independent experiments. The data were subjected to t-test. ${ }^{*} \mathrm{p}<0.05$. 
MiR-382 induce WB-F344 hepatic differentiation

To explore the effect of miR-382 on LPCs hepatic differentiation, the hepatocyte-specific markers (ALB, Ttr, ApoE) and liver stem marker (AFP) were detected by qPCR. Briefly, WB-F344 cells were transfected with miR-382 mimics, mimics negative control; miR-382 inhibitor, inhibitor negative control, respectively. qPCR analysis was performed at $24 \mathrm{~h}$ after transfection and Western Blotting analysis was performed at $48 \mathrm{~h}$ after transfection. As shown in Fig. 3, miR-382 mimics increased the expression of ApoE, Ttr, ALB, but decreased the level of AFP both in mRNA and protein level. These results indicate that miR-382 may induce WB-F344 hepatic differentiation.

\section{MiR-382 down-regulates Ezh2 directly}

To determine how miR-382 participates in the induction of hepatic differentiation, we sought to identify miR-382 target genes during hepatocytic differentiation. Bioinformatic tools (miRanda) for putative target genes were used and Ezh2 was identified by bioinformatic analysis. We then carried q RT-PCR and Western blotting analysis to determine whether miR382 mimics could regulate the expression of Ezh2. As shown in Fig. 4B and 4C, miR-382 drastically inhibited the expression of Ezh2 on both mRNA and protein levels. Conversely, miR-382 inhibitor significantly increased the level of Ezh2 both on protein and mRNA.

Fig. 3. miR-382 induce WB-F344 differentiation. A. Effect of miR-382 mimics or inhibitor on the mRNA of hepatocyte markers (ALB, Ttr, ApoE, AFP) in WBF344 cells. Data are shown as means and standard deviations from three independent experiments. The data were subjected to t-test. ${ }^{*} \mathrm{p}<0.05$. B. The protein level of AFP were determined by Western Blotting analysis. GAPDH was used as sample control. C. Statistical graph of Western Blotting bands. Data are expressed as mean \pm SD of three repeat experiments.

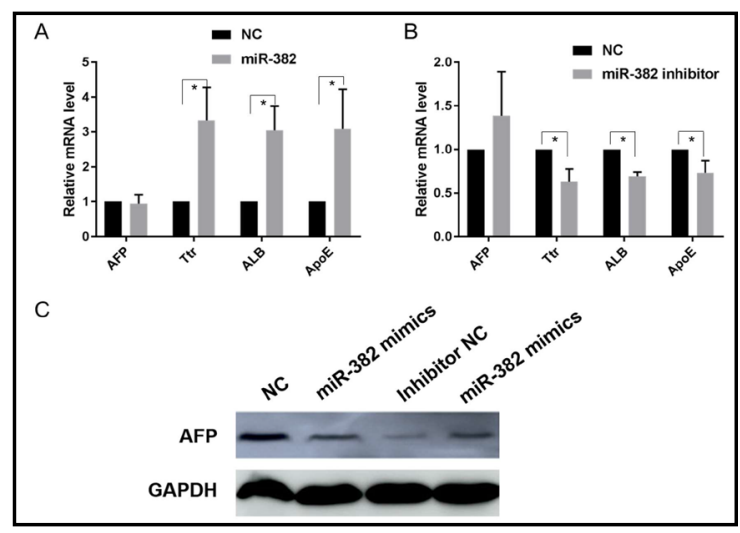

Fig. 4. Analyses of candidate target genes of miR-382. (A) Schematics indicating the interaction sites between the miR-382 and the 3'UTR of the Ezh2 mRNA. (B and C) WB-F344 cells transfected with miR-382 mimics or miR-382 inhibitor and their corresponding control were analyzed by qRT-PCR and Western Blotting analysis. GAPDH was used as control. (D) Dual luciferase assay of WBF344 cells cotransfected with the firefly luciferase constructs containing the various Ezh2 3'UTR, negative control and the miR382 mimics or negative control. Data are presented as the mean \pm standard error, based on at least three independent experiments. $* \mathrm{p}<0.05$.

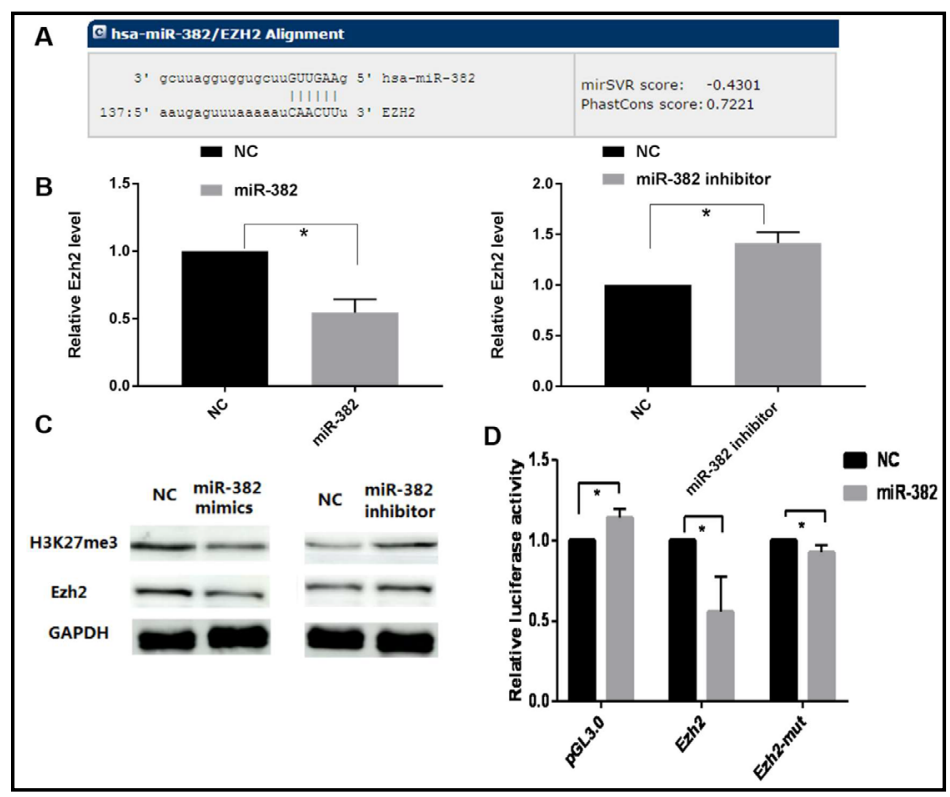




\section{Cellular Physiology Cell Physiol Biochem 2018;48:2389-2398 and Biochemistry \begin{tabular}{l|l} 
DOI: 10.1159/000492654 & (c) 2018 The Author(s). Published by S. Karger AG, Basel \\
www.karger.com/cpb
\end{tabular}

Fig. 5. Ezh2 downregulation promote hepatic differentiation in WB-F344 cells. A. Effect of Ezh2 siRNA on the expression of hepatocyte markers in WB-F344 cells were determined by qPCR. Data from three independent experiments are shown as the mean \pm standard error. B. The protein level of AFP was determined by Western Blotting analysis. C. Statistical graph of Western Blotting bands. Data are expressed as mean \pm SD of three repeat experiments.

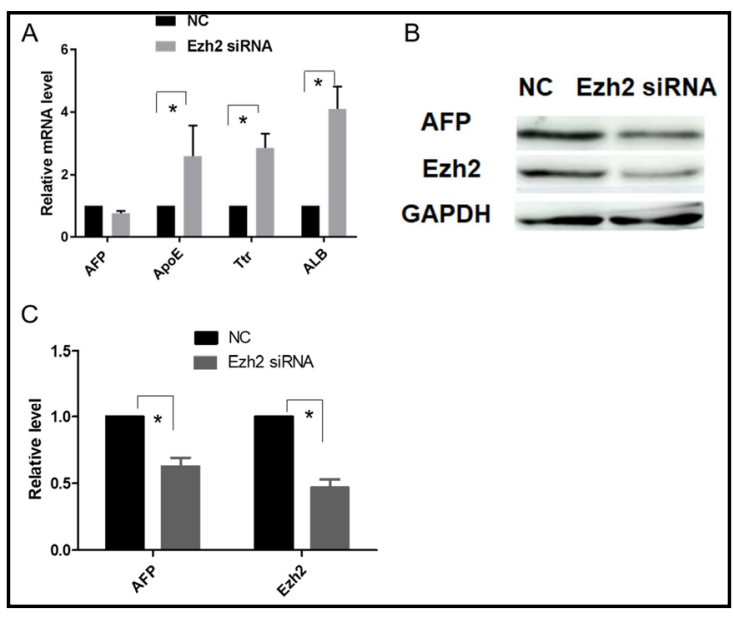

Furthermore, to determine whether Ezh2 was inhibited by miR-382 directly, the 3'UTR region of miR-382 was fused to a luciferase reporter (pmirGLO). As shown in Fig. 3D, miR382 repressed the luciferase activity of the wild-type plasmid (WT-Ezh2-pmirGLO) but not the mutant plasmid (Mu-Ezh2-pmirGLO), indicating that Ezh2 is a direct target of miR-382.

Knowing that Ezh2 can increase site-specific lysine methylation at H3K27 globally, we examined the H3K27me3 level in miR-382 treated WB-F344 cells. As shown in Fig. 3C, miR382 could induce up-regulation of global H3K27me3 level and Ezh2. These results indicate that miR-382 may regulate LPCs hepatic differentiation via the methylation modification of histone H3 mediated by Ezh2.

\section{Knockdown of Ezh2 promote WB-F344 hepatic differentiation}

As a major component of PRC2, Ezh2 mediates mesenchymal stem cell lineage specification [18] through chromatin modifications and contribute to the maintenance of self-renewal in a variety of stem cells. To confirm that the induction of hepatic differentiation by miR-382 was mediated by Ezh2, we assessed the effect of Ezh2 siRNA on hepatocyte differentiation. As presented in Fig. 5, Ezh2 siRNA not only increased mRNA level of hepatocyte-specific markers (ALB, Ttr, ApoE), but also increased the protein level of AFP. In other words, reduction of Ezh2 mimicked the effect of miR-382 overexpression. These results suggest that Ezh2 may be involved in the miR-382-mediated hepatic differentiation.

Furthermore, as revealed in Fig. 1C and Fig. 2B. We observed a significant down-regulation of Ezh2 on both the mRNA and protein levels during WB-F344 hepatic differentiation, in the opposite trend of miR-382. These data indicated an inverse correlation between the putative target genes Ezh2 and miR-382 expression during hepatocytic differentiation.

\section{Discussion}

The adult liver has a remarkable regenerative capacity, that can be utilized in clinical scenarios in living donor liver transplantation [25]. Recent evidences indicate that LPCs isolated from adult healthy mouse liver are important source of new mature hepatocytes for tissue maintenance and repair in vivo, and for regenerative medicine ex vivo [26, 27]. Understanding the mechanism that LPCs differentiation into hepatocytes may allow for novel induction strategies for chronic liver disease [28]. Here, we used rat liver progenitor cell WB-F344 as LPC cell model and HGF as inducer to simulate the process of LPCs hepatic differentiation [29]. Moreover, the hepatocyte cell trait was characterized in terms of hepatocellular function, hepatocellular morphology, and hepatocyte-specific markers [30].

Although several studies addressed the mechanism by which LPCs differentiated, the data is still insufficient to fully understand the mechanism of the well-ordered hepatic 


\section{Cellular Physiology Cell Physiol Biochem 2018;48:2389-2398 \begin{tabular}{l|l|l} 
DOI: 10.1159/000492654 & $\begin{array}{l}\text { O 2018 The Author(s). Published by S. Karger AG, Basel } \\
\text { www.karger.com/cpb }\end{array}$
\end{tabular}

differentiation. In particular, little is known about the roles of miRNAs and epigenetic factors in regulating hepatic differentiation. Knowing that miRNAs at the Dlk1-Dio3 imprinting locus are critical for the differentiation of stem cells [12] and the miR-379/656 sub-cluster can be activated by liver-specific hepatocyte nuclear factor4a (HNF4a) [31], implicating that Dlk1-Dio3 microRNA might be involved in the differentiation of LPC. In the present study, we identified several differentially expressed miRNAs in Dlk1-Dio3 microRNA cluster by q RTPCR. Then we confirmed that miR-382 was induced during WB-F344 hepatic differentiation and its higher expression in other hepatocyte cells than in WB-F344 cells, including rat liver tissues and BRL-3A cell line. Furthermore, enhanced expression of miR-382 increased the hepatocyte-specific markers. Therefore, we proposed that increased expression of miR382 may promote LPCs differentiation. Occasionally, according to previous reports, miR382 affect various lineage conversion, including adipocytes differentiation [32], osteogenic differentiation [33] and hematopoietic stem cell differentiation [34]. Together with our findings, we speculate that miR-382 is always involved in various stem cell differentiation.

To further investigate the specific role of miR-382 during WB-F344 hepatic differentiation, it is very important to identify miR-382 target genes. We used miRanda to predict the putative target genes of miR-382 and identified Ezh2, which was reported to inhibit hepatic differentiation of embryo liver, as direct target in WB-F344 cells. In our study, we found that miR-382 modulated H3K27me3 level by targeting Ezh2 in WB-F344 cells. Together with continuous decreased expression of Ezh2 and H3K27me3 during WBF344 hepatic differentiation, we considered that the induction of miR-382 may contribute to hepatic differentiation via a Ezh2-mediated methylation modification in histone H3 during WB-F344 hepatic differentiation.

In the 2-AAF/PH model, the oval cells were activated at earlier day and then differentiated into hepatocyte cells, which agree with previous study [23, 35]. Here, after 2-AAF/PH treatment, Ezh2 was firstly stimulated in the first 2 day and then decreased to an undetectable level in the following days, which coincided with increased expression of AFP. Generally, AFP was considered as the marker of oval cells, and could be used to monitor the oval cell activation and oval cell differentiation. According to our results, miR-382 was induced during LPCs differentiation. MiR-382 promote hepatic differentiation of WB-F344 by targeting Ezh2, which regulate the transcription repression of liver-specific genes. Our findings reveal a miRNA-mediated negative regulation pattern that occurs during LPCs hepatic differentiation and suggest an anti-proliferative role for miR-382 in liver cells.

\section{Acknowledgements}

The authors greatly appreciate the editors and the anonymous peer reviewers for the critical reading and insightful comments, which have improved our manuscript substantially. This study was supported by the National Natural Science Foundation of China (81400654) and China postdoctoral science foundation (2018M633092, 2017M612701).

\section{Disclosure Statement}

The authors have declared that no Disclosure Statement exists.

\section{References}

1 Zaret KS: Genetic programming of liver and pancreas progenitors: lessons for stem-cell differentiation. Nat Rev Genet 2008;9:329-340.

-2 Roskams T, De Vos R, Van Eyken P, Myazaki H, Van Damme B, Desmet V: Hepatic OV-6 expression in human liver disease and rat experiments: evidence for hepatic progenitor cells in man. J Hepatol 1998;29:455-463. 


\section{Cellular Physiology Cell Physiol Biochem 2018;48:2389-2398 \begin{tabular}{l|l|l} 
DOI: 10.1159/000492654 & (c) 2018 The Author(s). Published by S. Karger AG, Basel
\end{tabular} and Biochemistry Published online: 15 August, $2018 \quad$ www.karger.com/cpb}

3 Kuramitsu K, Sverdlov DY, Liu SB, Csizmadia E, Burkly L, Schuppan D, Hanto DW, Otterbein LE, Popov Y: Failure of fibrotic liver regeneration in mice is linked to a severe fibrogenic response driven by hepatic progenitor cell activation. Am J Pathol 2013;183:182-194.

4 Roskams T: Progenitor cell involvement in cirrhotic human liver diseases: from controversy to consensus. J Hepatol 2003;39:431-434.

5 Katoonizadeh A, Poustchi H: Adult Hepatic Progenitor Cell Niche: How it affects the Progenitor Cell Fate. Middle East J Dig Dis 2014;6:57-64.

6 Miyajima A, Tanaka M, Itoh T: Stem/progenitor cells in liver development, homeostasis, regeneration, and reprogramming. Cell Stem Cell 2014;14:561-574.

-7 Nibourg GA, Chamuleau RA, van der Hoeven TV, Maas MA, Ruiter AF, Lamers WH, Oude Elferink RP, van Gulik TM, Hoekstra R: Liver progenitor cell line HepaRG differentiated in a bioartificial liver effectively supplies liver support to rats with acute liver failure. PLoS One 2012;7:e38778. Bartel DP: MicroRNAs: genomics, biogenesis, mechanism, and function. Cell 2004;116:281-297. Altuvia Y, Landgraf P, Lithwick G, Elefant N, Pfeffer S, Aravin A, Brownstein MJ, Tuschl T, Margalit H: Clustering and conservation patterns of human microRNAs. Nucleic Acids Res 2005;33:2697-2706.

10 da Rocha ST, Edwards CA, Ito M, Ogata T, Ferguson-Smith AC: Genomic imprinting at the mammalian Dlk1Dio3 domain. Trends Genet 2008;24:306-316.

11 Liu L, Luo GZ, Yang W, Zhao X, Zheng Q Lv Z, Li W, Wu HJ, Wang L, Wang XJ, Zhou Q: Activation of the imprinted Dlk1-Dio3 region correlates with pluripotency levels of mouse stem cells. J Biol Chem 2010;285:19483-19490.

12 Lin SP, Coan P, da Rocha ST, Seitz H, Cavaille J, Teng PW, Takada S, Ferguson-Smith AC: Differential regulation of imprinting in the murine embryo and placenta by the Dlk1-Dio3 imprinting control region. Development 2007;134:417-426.

$\checkmark 13$ Cruz FM, Tome M, Bernal JA, Bernad A: miR-300 mediates Bmi1 function and regulates differentiation in primitive cardiac progenitors. Cell Death Dis 2015;6:e1953.

14 Mo CF, Wu FC, Tai KY, Chang WC, Chang KW, Kuo HC, Ho HN, Chen HF, Lin SP: Loss of non-coding RNA expression from the DLK1-DIO3 imprinted locus correlates with reduced neural differentiation potential in human embryonic stem cell lines. Stem Cell Res Ther 2015;6:1.

15 Cui L, Zhou X, Li J, Wang L, Wang J, Li Q, Chu J, Zheng L, Wu Q, Han Z, Shi Y, Han Y, Fan D: Dynamic microRNA profiles of hepatic differentiated human umbilical cord lining-derived mesenchymal stem cells. PLoS One 2012;7:e44737.

16 Surface LE, Thornton SR, Boyer LA: Polycomb group proteins set the stage for early lineage commitment. Cell Stem Cell 2010;7:288-298.

17 Sauvageau M, Sauvageau G: Polycomb group proteins: multi-faceted regulators of somatic stem cells and cancer. Cell Stem Cell 2010;7:299-313.

18 Aoki R, Chiba T, Miyagi S, Negishi M, Konuma T, Taniguchi H, Ogawa M, Yokosuka O, Iwama A: The polycomb group gene product Ezh2 regulates proliferation and differentiation of murine hepatic stem/progenitor cells. J Hepatol 2010;52:854-863.

19 Margueron R, Reinberg D: The Polycomb complex PRC2 and its mark in life. Nature 2011;469:343-349.

20 Koike H, Ouchi R, Ueno Y, Nakata S, Obana Y, Sekine K, Zheng YW, Takebe T, Isono K, Koseki H, Taniguchi H: Polycomb group protein Ezh2 regulates hepatic progenitor cell proliferation and differentiation in murine embryonic liver. PLoS One 2014;9:e104776.

21 Liu WH, Ren LN, Chen T, You N, Liu LY, Wang T, Yan HT, Luo H, Tang LJ: Unbalanced distribution of materials: the art of giving rise to hepatocytes from liver stem/progenitor cells. J Cell Mol Med 2014;18:1-14.

22 Wang X, Cui J, Zhang BQ Zhang H, Bi Y, Kang Q, Wang N, Bie P, Yang Z, Wang H, Liu X, Haydon RC, Luu HH, Tang N, Dong J, He TC: Decellularized liver scaffolds effectively support the proliferation and differentiation of mouse fetal hepatic progenitors. J Biomed Mater Res A 2014;102:1017-1025.

23 Hasuike S, Ido A, Uto H, Moriuchi A, Tahara Y, Numata M, Nagata K, Hori T, Hayashi K, Tsubouchi H: Hepatocyte growth factor accelerates the proliferation of hepatic oval cells and possibly promotes the differentiation in a 2-acetylaminofluorene/partial hepatectomy model in rats. J Gastroenterol Hepatol 2005;20:1753-1761.

-24 Gardiner E, Beveridge NJ, Wu JQ, Carr V, Scott RJ, Tooney PA, Cairns MJ: Imprinted DLK1-DIO3 region of 14q32 defines a schizophrenia-associated miRNA signature in peripheral blood mononuclear cells. Mol Psychiatry 2012;17:827-840. 


\section{Cellular Physiology Cell Physiol Biochem 2018;48:2389-2398 \begin{tabular}{ll|l} 
DOI: 10.1159/000492654 & $\begin{array}{l}\text { O 2018 The Author(s). Published by S. Karger AG, Basel } \\
\text { www.karger.com/cpb }\end{array}$
\end{tabular}

-25 Boulter L, Lu WY, Forbes SJ: Differentiation of progenitors in the liver: a matter of local choice. J Clin Invest 2013;123:1867-1873.

-26 Tanimizu N, Ichinohe N, Ishii M, Kino J, Mizuguchi T, Hirata K, Mitaka T: Liver Progenitors Isolated from Adult Healthy Mouse Liver Efficiently Differentiate to Functional Hepatocytes In vitro and Repopulate Liver Tissue. Stem Cells 2016;34:2889-2901.

-27 Du Y, Li D, Han C, Wu H, Xu L, Zhang M, Zhang J, Chen X: Exosomes from Human-Induced Pluripotent Stem Cell-Derived Mesenchymal Stromal Cells (hiPSC-MSCs) Protect Liver against Hepatic Ischemia/ Reperfusion Injury via Activating Sphingosine Kinase and Sphingosine-1-Phosphate Signaling Pathway. Cell Physiol Biochem 2017;43:611-625.

-28 Nicolas C, Wang Y, Luebke-Wheeler J, Nyberg SL: Stem Cell Therapies for Treatment of Liver Disease. Biomedicines 2016;4:pii:E2.

29 Yao P, Zhan Y, Xu W, Li C, Yue P, Xu C, Hu D, Qu CK, Yang X: Hepatocyte growth factor-induced proliferation of hepatic stem-like cells depends on activation of NF-kappaB. J Hepatol 2004;40:391-398.

-30 Jin S, Li H, Han M, Ruan M, Liu Z, Zhang F, Zhang C, Choi Y, Liu B: Mesenchymal Stem Cells with Enhanced Bcl-2 Expression Promote Liver Recovery in a Rat Model of Hepatic Cirrhosis. Cell Physiol Biochem 2016;40:1117-1128.

-31 Yin C, Wang PQ, Xu WP, Yang Y, Zhang Q, Ning BF, Zhang PP, Zhou WP, Xie WF, Chen WS, Zhang X: Hepatocyte nuclear factor-4alpha reverses malignancy of hepatocellular carcinoma through regulating miR-134 in the DLK1-DIO3 region. Hepatology 2013;58:1964-1976.

32 Choi WH, Ahn J, Jung CH, Jang YJ, Ha TY: beta-Lapachone Prevents Diet-Induced Obesity by Increasing Energy Expenditure and Stimulating the Browning of White Adipose Tissue via Downregulation of miR382 Expression. Diabetes 2016;65:2490-2501.

-33 Heilmeier U, Hackl M, Skalicky S, Weilner S, Schroeder F, Vierlinger K, Patsch JM, Baum T, Oberbauer E, Lobach I, Burghardt AJ, Schwartz AV, Grillari J, Link TM: Serum miRNA Signatures Are Indicative of Skeletal Fractures in Postmenopausal Women With and Without Type 2 Diabetes and Influence Osteogenic and Adipogenic Differentiation of Adipose Tissue-Derived Mesenchymal Stem Cells In vitro. J Bone Miner Res 2016;31:2173-2192.

34 Zini R, Rossi C, Norfo R, Pennucci V, Barbieri G, Ruberti S, Rontauroli S, Salati S, Bianchi E, Manfredini R: miR-382-5p Controls Hematopoietic Stem Cell Differentiation Through the Downregulation of MXD1. Stem Cells Dev 2016;25:1433-1443.

35 Xiang S, Chen XP, Zhang W, Zhang BH, Liang HF: [A study of oval cell proliferation kinetics in the rat 2-acetylaminofluorene/partial hepatectomy model]. Zhonghua Wai Ke Za Zhi 2010;48:615-618. 